\title{
The D-dimer level predicts the prognosis in patients with lung cancer: a systematic review and meta-analysis
}

\author{
Mingsheng Ma ${ }^{1,2+}$, Run Cao ${ }^{1 \dagger}$, Wei Wang ${ }^{1}$, Biying Wang ${ }^{1}$, Yichen Yang ${ }^{1}$, Yunchao Huang ${ }^{1}$, \\ Guangqiang Zhao ${ }^{1}$ and Lianhua Ye $\mathrm{i}^{\text {* }^{*}}$
}

\begin{abstract}
Objective: Although the significance of increased plasma D-dimer levels in activating coagulation and fibrinolysis has been reported, it is still controversial whether it can be used to predict the prognosis of lung cancer patients. This meta-analysis was performed to explore the beneficial role of plasma D-dimer as a prognostic factor in lung cancer patients according to a larger sample capacity.
\end{abstract}

Materials and methods: MEDLINE, EMBASE, and Cochrane Central databases were searched from inception to January 2021. The data are mainly hazard ratio(HR) with 95\% confidence interval (CI) and Kaplan-Meier survival curves. The publication bias was examined by Egger's test.

Results: Finally, a total of 28 studies, enrolling 8452 patients were included in the current meta-analysis. Our results showed that the OS $(\mathrm{HR}=1.742,95 \% \mathrm{Cl}: 1.542-1.969, P<0.001)$ and $\mathrm{PFS}(\mathrm{HR}=1.385,95 \% \mathrm{Cl}: 1.169-1.641, P=0.003)$ in the high D-dimer group were significantly lower than those in the low D-dimer group. Subgroup analysis suggested that localization, detection methods and disease stage had an important effect on the prognosis.

Conclusion: This meta-analysis revealed that the high plasma D-dimer level leads to lower survival than in the low D-dimer level, which might provide an important clue for high plasma D-dimer level as an independent factor of poor prognosis in patients with lung cancer.

Keywords: D-dimer, Prognosis, Lung cancer

\section{Introduction}

Lung cancer has become the most common malignant tumor and the leading cause of cancer death in the world. Activation of coagulation and fibrinolysis is usually associated with most malignant tumors [1], although the exact molecular mechanism remains incompletely understood. Previous studies have found that malignancy

\footnotetext{
*Correspondence: Ihye1204@aliyun.com

${ }^{\dagger}$ Mingsheng Ma and Run Cao have contributed equally to this work. ${ }^{1}$ Department of Thoracic Surgery, The Third Affiliated Hospital of Kunming Medical University, No. 519 Kunzhou Road, Xishan District, Kunming City, Yunnan Province, China

Full list of author information is available at the end of the article
}

can affect the hemostatic system; however, the activation of the hemostatic system can influence the biological behavior of tumors [2]. The coagulation and fibrinolysis system activation can accelerate the growth and invasion of tumor cells, thus affecting cancer progression [3]. Lung cancer patients reveal an abnormal coagulation state, including venous thromboembolism (VTE) [4]. Tumorinduced thrombosis has a remarkable effect on the prognosis of patients with cancer.

Plasma D-dimer is the lysis end-product of crosslinked fibrin protein degradation and is a critical forecast indicator of coagulation dysfunction [5]. Plasma D-dimer levels increase by promoting fibrin formation original author(s) and the source, provide a link to the Creative Commons licence, and indicate if changes were made. The images or other third party material in this article are included in the article's Creative Commons licence, unless indicated otherwise in a credit line to the material. If material is not included in the article's Creative Commons licence and your intended use is not permitted by statutory regulation or exceeds the permitted use, you will need to obtain permission directly from the copyright holder. To view a copy of this licence, visit http://creativecommons.org/licenses/by/4.0/. The Creative Commons Public Domain Dedication waiver (http://creativecommons.org/publicdomain/zero/1.0/) applies to the data made available in this article, unless otherwise stated in a credit line to the data. 
and fibrinolysis. In the past few years, plasma D-dimer elevated levels have been attracted much attention with different malignant tumors, including colorectal cancer, gastric colorectal, cervical, breast, esophagus cancer $[6,7]$. Plasma D-dimer levels have played a vital role in excluding thrombosis associated with clinical cancer [8]. In these studies, a high D-dimer level was found and associated with the prognosis of the patients. Therefore, the plasma D-dimer level can be used as an effective prognostic predictor.

D-dimer elevated levels have also been found in lung cancer patients and associated with a poor prognosis $[9,10]$. It has been reported that tumors with a higher angiogenesis and metastasis manner biologically degree behave related to activating the coagulation system [11]. However, these analyzed studies are only based on data such as certain quantities of small cell lung cancer (SCLC) patients and tumor staging (I-IV). The prognostic value of D-dimer in lung cancer remains limited. Although many studies demonstrated that a high D-dimer level is related to the prognosis of lung cancer, the plasma D-dimer levels as a lung cancer prognostic criterion are still controversial.

In this study, we performed a meta-analysis of collecting current comparative data to further determine D-dimer level's prognostic significance in patients with lung cancer.

\section{Methods}

\section{Search strategy and eligibility criteria}

We performed a literature search through the following databases: Pubmed, Cochrane Central databases, Web of Science and EMBASE databases for studies published before January 2021. The keywords we defined were as follows: "lung cancer," "D-dimer," and "prognosis" and the search strategy were used: ("lung cancer" OR "Pulmonary Neoplasm" OR "Lung Neoplasm") AND ("D-dimer" OR "fibrinolysis") AND ("prognosis" OR "prognostic"). The references of relevant studies and review articles were also checked to identify additional studies. Two authors (MM and WW) assessed the titles and abstracts independently to extract the full articles for all potentially relevant studies from each eligible report. This systematic review with individual patient data meta-analysis was registered on INPLASY (INPLASY202170096).

All retrieved articles included observational studies assessing the inclusion and exclusion criteria described. The selected studies inclusion criteria were as follows: 1) patients with lung cancer including any treatments; 2) articles investigating the correlation of D-dimer levels which collected before treating with the survival of lung cancer; 3) overall survival (OS) and relapse-free survival (PFS); 4) data: the necessary survival data must be provided, including hazard ratio (HR), 95\% confidence interval (CI) and Kaplan-Meier curve; 5) full text is available. The following criteria were excluded: 1) records were not written in English; 2) records such as abstracts, letters, reviews, case reports, or nonclinical studies; 3 ) full text is unavailable; and 4) follow-up time less than 5 years.

\section{Data extraction and quality assessment}

The following data were performed by the two authors (BW and YY) independently. The information should be extracted included:(1)author name, publication year, sample size, age, gender, median follow-up time; (2)disease stage, treatment approach, histology type, detection method, location; (3) the risk ratio (HR) and their associated $95 \% \mathrm{CI}$, The primary prognosis outcomes were OS and PFS. If the title and abstract cannot be classified, the full text should be read. Two authors (YH and GZ) evaluated the quality of studies independently if disagreement occurred, and the third investigator made the final agreed decision (LY). The value was assessed through methods in the literature if the HR was not given directly [12, 13]. The quality of the studies was evaluated in accordance with the Newcastle-Ottawa Scale (NOS). NOS contained three domains: patient selection (0-4 points), comparability ( $0-2$ points), and outcome ( $0-3$ points). NOS scores ranged from 0 to 9 points, and studies with an NOS score $\geq 6$ were considered to be of high quality.

\section{Statistical analysis}

The association of D-dimer with OS and PFS was evaluated by pooling HRs and 95\% CIs. Heterogeneity was assessed with Chi-square and the $\mathrm{I}^{2}$ index. A P-value $\geq 0.1$ and $\mathrm{I}^{2}<50 \%$ were considered not statistically significant, and the fixed effects model was used. A P-value $<0.1$ and $\mathrm{I}^{2} \geq 50 \%$ was regarded as high heterogeneity. The random-effected model was chosen to pool the heterogeneous studies [14]. Publication bias was used to assess by Begg's funnel plot. We used STATA version 15.1 to perform the meta-analysis. All statistical tests were two-tailed, and $P<0.05$ was set statistically significant.

\section{Results}

\section{Study and patient characteristics}

We screened 1593 studies from the searches. After carefully testing these articles, a total of 28 studies, including 8452 patients published between 1997 and 2021, were included in the meta-analysis. In this analysis, the high D-dimer group was chosen as the reference. All included studies were retrospective. The flow diagram of the detailed process of study selection is shown in Fig. 1. Among them, 4 studies were from Japan, 15 studies were performed in China, 3 studies were from Italy, 5 studies 

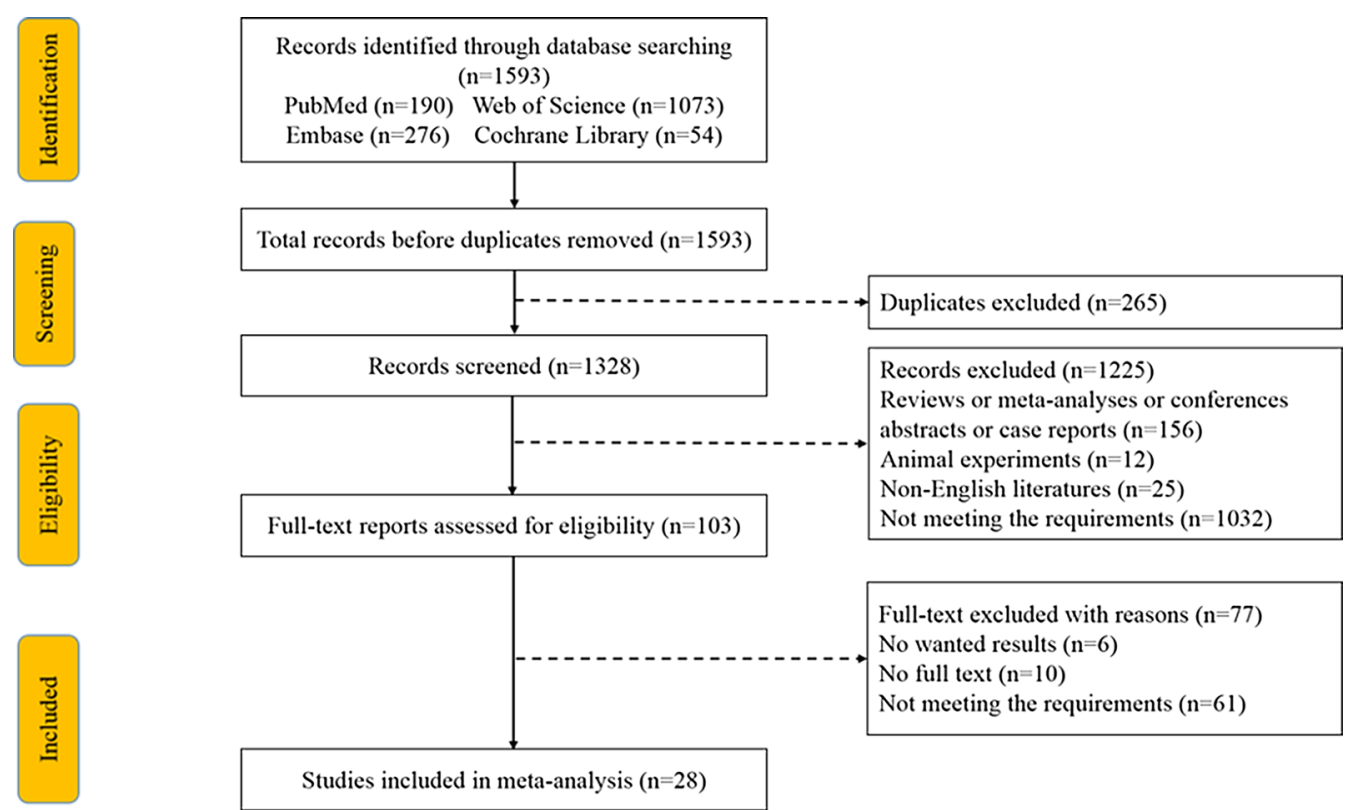

Studies included in meta-analysis $(\mathrm{n}=\mathbf{2 8})$

Fig. 1 The flow diagram of literature search

were from Turkey, 1 study was from Australia, Austria, and Russia, 27 studies used OS as a unique prognostic indicator, 7 studies described PFS. The characteristics of the enrolled studies are summarized in Table 1. All patients included had a stage I-IV disease and received different treatments. The median age of these patients was 57 to 62 years old. All but five studies were followed up for a relatively long time. However, some studies have different cut-off values. The HR and $95 \% \mathrm{CI}$ were obtained directly or indirectly through multivariate Cox regression analysis.

\section{Quality assessment and risk of bias}

All studies were retrospective cohort studies, and we evaluated the quality based on the modified NOS scale. The results of the quality assessment were listed in Table 1. All studies are considered to be of high quality, indicating that the risk of bias in our entire study is very low.

\section{Meta-analysis of the D-dimer level on OS and PFS with lung cancer patients}

Twenty-seven studies included 5,455 patients compared OS between lung cancer patients with high D-dimer level and these with normal D-dimer level. Our metaanalysis found that the OS of lung cancer patients with high D-dimer levels was worse than that of lung cancer patients with normal D-dimer level (random effects: $\mathrm{HR}=1.742$, 95\% CI:1.542-1.969; $P<0.001, \mathrm{I}^{2}=72.1 \%$ ) (Fig. 2a). Seven studies included 2997 patients compared
PFS between patients with high D-dimer level and those with normal D-dimer level. Our meta-analysis found that lung cancer patients with high D-dimer level also had a significantly worse PFS (random effects: $\mathrm{HR}=1.385,95 \%$ CI:1.169-1.641; $P=0.003, \mathrm{I}^{2}=69.1 \%$ ) (Fig. 2b).

\section{Subgroup analysis Detection methods}

The detection methods used in the studies include immunoturbidimetry assay [15-27], latex assay [1, 28-30], and enzyme-linked immunosorbent assay (ELISA) [9, 31-36]. Since different detection methods may affect the level of D-dimer, we conducted a subgroup analysis to further analyze the impact of different detection methods on the prognosis. The HRs and 95\%CI for OS in immunoturbidimetry group assay was 1.363 (1.264-1.470), in group latex assay were $1.513(1.266-1.810)$, and in the group ELISA was 2.144(1.466-3.134) ( Additional file 1: Figure S1 a-c). The HR and 95\% CI for PFS in immunoturbidimetry groups assay was 1.461 (1.148-1.861), in group latex assay were 1.340 (1.167-1.539) (Additional file 2: Figure S2 a-b).

\section{Histological type}

95\% CI we also divided the studies into histological types (adenocarcinoma / total $>25 \%$ or $<25 \%$ ). We found that the HR and $95 \% \mathrm{CI}$ of OS in $>25 \%$ adenocarcinoma group were 1.639 (1.298-2.069), and 1.976 (1.651- 2.364) for $<25 \%$ adenocarcinoma (Additional file 3: Figure S3ab). In the adenocarcinoma $>25 \%$ group, the $H R$ and 
Table 1 Summary table of the meta-analysis

\begin{tabular}{|c|c|c|c|c|c|c|c|c|c|c|c|}
\hline Auther(year) & Country & \multicolumn{2}{|c|}{$\begin{array}{l}\text { Samplesize(N) } \\
\text { (F/M) }\end{array}$} & \multicolumn{2}{|c|}{ Cutoff value } & Age (range) & $\begin{array}{l}\text { Follow-up } \\
\text { (median) }\end{array}$ & $\begin{array}{l}\text { Detection } \\
\text { method }\end{array}$ & \multicolumn{2}{|c|}{ Outcomes } & $\begin{array}{l}\text { Quality } \\
\text { assessment }\end{array}$ \\
\hline Buccheri et al. [17] & Italy & \multicolumn{2}{|c|}{ 286(31/255) } & \multicolumn{2}{|c|}{$0.5 \mathrm{~g} / \mathrm{mL}$} & $61(32-87)$ & 34 months & Latex assay & \multicolumn{2}{|l|}{ OS } & 7 \\
\hline Taguchi et al. [18] & Japan & \multicolumn{2}{|c|}{70} & \multicolumn{2}{|c|}{$150 \mathrm{ng} / \mathrm{ml}$} & $65(20-83)$ & 15 months & ELISA & \multicolumn{2}{|l|}{ OS } & 5 \\
\hline Ferrigno et al. [19] & Italy & \multicolumn{2}{|c|}{$343(31 / 304)$} & \multicolumn{2}{|c|}{$0.5 \mathrm{ug} / \mathrm{ml}$} & $68(39-86)$ & - & $\begin{array}{l}\text { Immunoturbidim- } \\
\text { etry }\end{array}$ & \multicolumn{2}{|l|}{ OS } & 8 \\
\hline Pavey et al. [20] & Australia & \multicolumn{2}{|c|}{ 166(38/128) } & \multicolumn{2}{|c|}{$32.99 \mathrm{ng} / \mathrm{ml}$} & $64(30-86)$ & 270 days & ELISA & \multicolumn{2}{|l|}{ OS } & 8 \\
\hline Buccheri et al. [21] & Italy & \multicolumn{2}{|c|}{$826(99 / 727)$} & \multicolumn{2}{|c|}{$0.5 \mathrm{ug} / \mathrm{ml}$} & $67(35-89)$ & 34 weeks & $\begin{array}{l}\text { Immunoturbidim- } \\
\text { etry }\end{array}$ & \multicolumn{2}{|l|}{ OS } & 8 \\
\hline Altiay et al. [23] & Turkey & \multicolumn{2}{|c|}{$78(5 / 73)$} & \multicolumn{2}{|c|}{$0.65 \mathrm{ug} / \mathrm{ml}$} & $61(37-82)$ & 264 days & ELISA & \multicolumn{2}{|l|}{ OS } & 8 \\
\hline $\begin{array}{l}\text { Komurcuoglu et al. } \\
\text { [24] }\end{array}$ & Turkey & \multicolumn{2}{|c|}{$100(14 / 86)$} & \multicolumn{2}{|c|}{$1250 \mathrm{ng} / \mathrm{dl}$} & 67 & - & ELISA & \multicolumn{2}{|l|}{ OS } & 6 \\
\hline Masago et al. [25] & Japan & \multicolumn{2}{|c|}{$99(28 / 71)$} & \multicolumn{2}{|c|}{$0.6 \mathrm{ng} / \mathrm{ml}$} & $72(35-88)$ & $0-800$ days & ELISA & \multicolumn{2}{|l|}{ OS } & 8 \\
\hline Ay et al. [26] & Austria & 182 & & 0.8 & $\mathrm{~g} / \mathrm{mL}$ & $62(52-68)$ & 731 days & Latex assay & OS & & 7 \\
\hline Tas et al. [2] & Turkey & $110(1$ & /100) & 360 & IU/ml & $59(35-80)$ & 20.3 weeks & $\begin{array}{l}\text { Microparticle } \\
\text { Enzyme Immu- } \\
\text { noassay }\end{array}$ & OS & & 7 \\
\hline Zhang et al. [27] & China & $232(8$ & /149) & 0.3 & $\mu \mathrm{g} / \mathrm{ml}$ & $61(30-86)$ & 47.0 months & $\begin{array}{l}\text { Immunoturbidim- } \\
\text { etry }\end{array}$ & OS & & 6 \\
\hline $\begin{array}{l}\text { Fukumoto et al. } \\
\text { [28] }\end{array}$ & Japan & $237(8$ & /152) & $0.5 c$ & $\lg / \mathrm{ml}$ & $69(31-85)$ & 51.6 months & - & OS & & 7 \\
\hline Ursavaş et al. [29] & Turkey & $65(10$ & & 375 & $\mu \mathrm{g} / \mathrm{L}$ & 60 & $0-1,000$ days & Latex assay & OS & & \\
\hline Ge et al. [30] & China & $82(27$ & & - & & $64(44-72)$ & - & $\begin{array}{l}\text { Immunoturbidim- } \\
\text { etry }\end{array}$ & OS/ & & 8 \\
\hline Inal et al. [9] & Turkey & $72(16$ & & 190 & $0 \mathrm{ng} / \mathrm{mL}$ & - & 574.14 days & ELISA & OS & & 8 \\
\hline Wang et al. [31] & China & 1929 & $04 / 1325)$ & 0.5 & $\mathrm{ag} / \mathrm{mL}$ & - & 18.0 months & $\begin{array}{l}\text { Immunoturbidim- } \\
\text { etry }\end{array}$ & OS/ & & 7 \\
\hline Chen et al. [22] & China & $393(7$ & /322) & 0.5 & $\mathrm{ug} / \mathrm{ml}$ & $57(49-65)$ & 12 months & $\begin{array}{l}\text { Immunoturbidim- } \\
\text { etry }\end{array}$ & OS/ & & 6 \\
\hline Zhu et al. [32] & China & $74(17$ & & 0.5 & $u g / L$ & $57(42-80)$ & 11.5 months & $\begin{array}{l}\text { Immunoturbidim- } \\
\text { etry }\end{array}$ & OS/ & & 8 \\
\hline Jiang [33] & China & $107(2$ & /84) & 0.5 & $\mathrm{mg} / \mathrm{L}$ & $63.0(58.5,68.0)$ & 9 months & $\begin{array}{l}\text { Immunoturbidim- } \\
\text { etry }\end{array}$ & OS & & 8 \\
\hline Sun [18] & China & $272(1$ & 9/163) & 0.5 & $\mathrm{mg} / \mathrm{L}$ & 65 & - & - & OS & & 6 \\
\hline Zhang et al. [34] & China & $160(3$ & /129) & 500 & $\mathrm{ng} / \mathrm{ml}$ & $59(23-83)$ & - & $\begin{array}{l}\text { Immunoturbidim- } \\
\text { etry }\end{array}$ & PFS & & 8 \\
\hline Fan et al. [35] & China & $82(15$ & & 0.5 & $\mathrm{mg} / \mathrm{L}$ & $60(28-82)$ & $0-50$ months & $\begin{array}{l}\text { Immunoturbidim- } \\
\text { etry }\end{array}$ & PFS, & & 8 \\
\hline Hou et al. [36] & China & $395(1$ & $0 / 225)$ & 0.2 & $\mathrm{mg} / \mathrm{L}$ & $64(56-69)$ & 13.2 months & $\begin{array}{l}\text { Immunoturbidim- } \\
\text { etry }\end{array}$ & OS & & 6 \\
\hline Liang et al. [37] & China & $456(1$ & 8/318) & 500 & $\mathrm{ng} / \mathrm{ml}$ & $61(35-81)$ & 42 months & $\begin{array}{l}\text { Immunoturbidim- } \\
\text { etry }\end{array}$ & OS & & 8 \\
\hline Shiina et al. [38] & Japan & $235(8$ & /146) & 1.0 & $\mathrm{ug} / \mathrm{ml}$ & 70 & $0-3$ years & - & OS & & 8 \\
\hline Chen et al. [15] & China & $233(7$ & /1630) & 500 & $\mathrm{mg} / \mathrm{ml}$ & 67 & 0-60 months & ELISA & OS & & 7 \\
\hline Liu et al. [16] & China & $651(2$ & 6/435) & 0.5 & $\mathrm{ng} / \mathrm{L}$ & 60 & 0-80 months & $\begin{array}{l}\text { Immunoturbidim- } \\
\text { etry }\end{array}$ & OS & & 7 \\
\hline Moik et al. [39] & Russia & $277(1$ & 3/174) & $1 \mathrm{~m}$ & $\mathrm{~g} / \mathrm{dl}$ & $61(56-67)$ & 24 months & Latex assay & OS/ & & 8 \\
\hline Auther(year) & Treatmen & & & & & & & Histology typ & & Disea & se stage ( $\mathrm{TO} /$ \\
\hline & $\begin{array}{l}\text { Chemoth } \\
\text { (alone/ } \\
\text { combinat }\end{array}$ & $\begin{array}{l}\text { erapy } \\
\text { tion) }\end{array}$ & Radiot & & $\begin{array}{l}\text { Chemo- } \\
\text { radiation }\end{array}$ & Surgery & $\begin{array}{l}\text { No anti } \\
\text { treatme } \\
\text { support }\end{array}$ & & & & \\
\hline Buccheri et al. [17] & - & & - & & & - & - & $\begin{array}{l}\mathrm{E} / \mathrm{S} / \mathrm{A} / \mathrm{L} / \mathrm{U} \\
112 / 37 / 67 / 1\end{array}$ & $8 / 52$ & $\begin{array}{r}|/||/||| / \mid \\
42 / 3\end{array}$ & $\begin{array}{l}\text { IV } \\
31 / 119 / 94\end{array}$ \\
\hline Taguchi et al. [18] & 50 & & 20 & & 0 & $\begin{array}{l}\text { NSCLC 49; SC } \\
21\end{array}$ & - & & & & \\
\hline
\end{tabular}


Table 1 (continued)

\begin{tabular}{|c|c|c|c|c|c|c|c|}
\hline \multirow[t]{2}{*}{ Auther(year) } & \multicolumn{5}{|l|}{ Treatment } & \multirow[t]{2}{*}{ Histology type } & \multirow{2}{*}{$\begin{array}{l}\text { Disease stage (T0/ } \\
\mathrm{T} 1 / \mathrm{T} 2 / \mathrm{T} 3 / \mathrm{T} 4)\end{array}$} \\
\hline & $\begin{array}{l}\text { Chemotherapy } \\
\text { (alone/ } \\
\text { combination) }\end{array}$ & Radiotherapy & $\begin{array}{l}\text { Chemo- } \\
\text { radiation }\end{array}$ & Surgery & $\begin{array}{l}\text { No anticancer } \\
\text { treatment or } \\
\text { supportive care }\end{array}$ & & \\
\hline Ferrigno et al. [19] & 175 & 8 & - & 66 & $26 \%$ & $\begin{array}{l}E / A / S / L / U \\
125 / 80 / 34 / 16 / 88\end{array}$ & $\begin{array}{l}|/||| / \mid I / / V \\
69 / 26 / 124 / 121\end{array}$ \\
\hline Pavey et al. [20] & - & - & - & - & - & $\begin{array}{l}\text { NSCLC 166(A/E/L/O } \\
68 / 76 / 13 / 9)\end{array}$ & - \\
\hline Buccheri et al. [21] & & & & & & $\begin{array}{l}\text { E/S/A/L/U } \\
\text { 296/93/210/48/179 }\end{array}$ & $\begin{array}{l}|/ /| 1 /|| / / V \\
9 / 149 / 57 / 310 / 305\end{array}$ \\
\hline Altiay et al. [23] & 74 & 57 & & - & 4 & NSCLC 60; SCLC 18 & III/IV 35/43 \\
\hline $\begin{array}{l}\text { Komurcuoglu } \\
\text { et al. [24] }\end{array}$ & - & - & & - & - & NSCLC 87; SCLC 13 & II/III/IV 15/50/35 \\
\hline Masago et al. [25] & 67 & 2 & 8 & - & 22 & A/E/N 68/18/13 & III/IV42/57 \\
\hline Ay et al. [26] & - & - & - & - & - & - & - \\
\hline Tas et al. [2] & & & & & & $\begin{array}{l}\text { E/A/U/S } \\
\text { 26/30/28/16 }\end{array}$ & \\
\hline Zhang et al. [27] & - & - & - & 232 & - & E/A 111/121 & $|-||/||| 173 / 59$ \\
\hline $\begin{array}{l}\text { Fukumoto et al. } \\
\text { [28] }\end{array}$ & - & - & - & 237 & - & $\begin{array}{l}\text { E/A/L/O } \\
51 / 162 / 7 / 17\end{array}$ & - \\
\hline Ursavaş et al. [29] & - & - & - & - & - & E/A/S/U 32/11/17/5 & I/II/III/IV 20/5/16/32 \\
\hline Ge et al. [30] & 82 & - & - & - & - & N-SCC/SCC 53/29 & IIIB/IV 10/72 \\
\hline Inal et al. [9] & 72 & - & - & - & - & $\begin{array}{l}\text { E/A/N/S } \\
19 / 14 / 24 / 15\end{array}$ & III/IV 33/22 \\
\hline Wang et al. [31] & - & - & - & - & - & A/O 1046/885 & IV1929 \\
\hline Chen et al. [22] & 126 & - & 267 & - & - & SCLC 393 & - \\
\hline Zhu et al. [32] & 74 & - & - & - & - & SCLC 74 & - \\
\hline Jiang [33] & 25 & - & 82 & - & - & SCLC 107 & - \\
\hline Sun [18] & 177 & - & - & - & - & N-SCLC 272 & IV 272 \\
\hline Zhang et al. [34] & 160 & - & - & - & - & SCLC 160 & LD/ED 38/122 \\
\hline Fan et al. [35] & 27 & - & 51 & - & - & SCLC 82 & $\begin{array}{l}|+||+||| a /|| \mid b+I V \\
19 / 63\end{array}$ \\
\hline Hou et al. [36] & - & - & - & 395 & - & $\begin{array}{c}\mathrm{NSCLC}(\mathrm{E} / \mathrm{A} / \mathrm{O}) \\
53 / 329 / 13\end{array}$ & $|-||/||| \mid 341 / 54$ \\
\hline Liang et al. [37] & - & - & - & 456 & - & NSCLC 61 & |/II/II 170/101/185 \\
\hline Shiina et al. [38] & 46 & 2 & 9 & 235 & - & $\begin{array}{l}\text { Adenocarcinoma/ } \\
\text { Squamous cell } \\
\text { carcinoma/ } \\
\text { LCNEC/Large } \\
\text { cell carcinoma/ } \\
\text { Pleomorphic car- } \\
\text { cinoma/Carcinoid } \\
\text { 186/55/2/2/2/2 } \\
\text { A/E/L/L/P/C } \\
\text { 186/55/2/2/2/2 }\end{array}$ & - \\
\hline Chen et al. [15] & - & - & - & - & - & E/A/O 55/124/54 & III/IV 110/123 \\
\hline Liu et al. [16] & 492 & 70 & 89 & $\begin{array}{l}\text { E/A/O } \\
126 / 504 / 21\end{array}$ & III/IV98/553 & & \\
\hline Moik et al. yy[39] & 277 & 277 & - & 277 & - & $\begin{array}{l}\text { NSCLC/SCLC } \\
231 / 46\end{array}$ & |/II/III/IV 1/4/76/196 \\
\hline
\end{tabular}




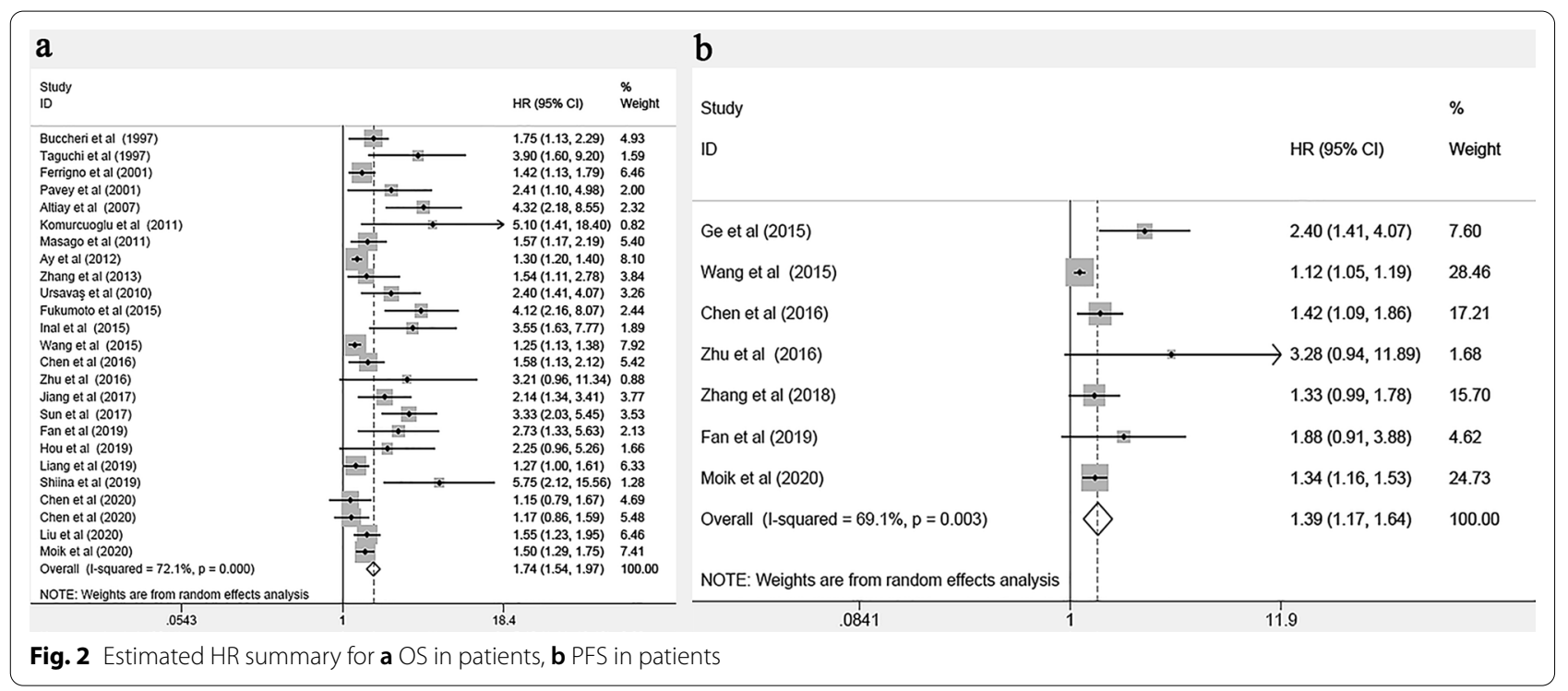

95\%CI of PFS was 1.120(1.052,1.192), and those of adenocarcinoma $<25 \%$ were 1.461 (1.148-1.861) (Additional file 4: Figure S4 a-c).

\section{Disease stage}

We also divided the studies into clinical stage (stage IIIIV/total $>80 \%$ or $<80 \%$ ). The HRs and $95 \% \mathrm{CI}$ for OS in group stage III-IV/total $>80 \%$ was $1.681(1.377-2.053)$, in group stage III-IV/total $<80 \%$ was $1.352(1.265-1.445)$ Additional file 5: Figure S5 a-c. The HRs and 95\% CI for PFS in group stage III-IV/total $>80 \%$ was $1.431(1.022-$ $2.004)$, in group stage III-IV/total $<80 \%$ was 2.400 (1.413-4.078) (Additional file 6: Figure S6 a-c).

\section{Other groups}

Three reports originated from Europe, Twenty from Asia, and two from Oceania. When we subgrouped the analysis by patients' resources, the HRs and 95\% CI for OS in patients in Asia 1.979 (1.646-2.39), Europe were 1.504(1.335-1.695) and Oceania were 1.572 (0.8992.746) (Additional file 7: Figure S7 a-c), respectively. The HRs and 95\% CI for PFS in patients in Asia 1.461 (1.148, 1.861, Europe were 1.340(1.167-1.539) (Additional file 8: Figure S8 a-b). We also divided the studies into different treatments (non-surgical, surgical, or mixed and found that the HRs and 95\% CI for OS in the non-surgical group were $2.380(1.780-3.0)$ the surgical group was 1.910 (1.17-3.14) and for and mixed group 1.688 (1.333-2.139) (Additional file 9: Figure S9 a-b). The HRs and 95\% CI for PFS in the non-surgical group were 310 (1.076-1.596) and mixed group 2.400 (1.413-4.078) (Additional file 10: Figure S10 a-b).
The results of meta-analyses of prediction value for overall and subgroup analysis were shown in Table 2.

\section{Sensitivity analysis and publication bias}

The stability of OS and PFS was evaluated by sensitivity analysis. Sensitivity analysis is to remove each study in turn to observe whether to change the original overall analysis results, the results show that each study deleted in turn did not change the original results, indicating that the results are more reliable (Fig. 3a, 3b). Publication bias was tested by Begg's test, and the results showed that OS had no publication bias $(P=0.871)$ (Fig. 4$)$.

\section{Discussion}

As we all know, lung cancer has been widely concerned and studied by the society because of its high mortality rate. In recent years, due to the rapid development of surgical technology, various treatments including radiotherapy and chemotherapy have greatly improved the survival of lung cancer patients. However, we believe that to improve the survival rate and quality of life of lung cancer patients, it is necessary to find a way to predict prognostic survival as soon as possible. Therefore, many researchers are looking for biomarkers that can predict the prognosis of lung cancer, and use this to provide guidance for the later clinical treatment of lung cancer.

$\mathrm{D}$-dimer is involved in the regulation of multiple cancer processes, so in recent years, there have been many studies exploring the relationship between D-dimer levels and the prognosis of lung cancer patients [15, 31]. Han proved that high D-dimer level is associated with the danger of occult tumor in patients with unprovoked venous thromboembolism (VTE) [37]. In cancer 
Table 2 results of meta-analyses of prediction value

\begin{tabular}{|c|c|c|c|c|c|}
\hline Survival outcome & No & $\mathrm{HR}[95 \% \mathrm{Cl}]$ & Log-rank $p$ & Heterogeneity $(p, 12(\%))$ & Publication bias \\
\hline OS & 27 & $1.742(1.542,1.969)$ & $<0.001$ & $<0.001,72.1 \%$ & 0.871 \\
\hline \multicolumn{6}{|l|}{ location } \\
\hline Asia & 20 & $1.979(1.646,2.379)$ & $<0.001$ & $<0.001,75.3 \%$ & \\
\hline Europe & 3 & $1.504(1.335,1.695)$ & $<0.001$ & $0.623,0.0 \%$ & \\
\hline Oceania & 2 & $1.572(0.899,2.746)$ & 0.112 & $0.111,60.6$ & \\
\hline \multicolumn{6}{|c|}{ Histology type (adenocarcinoma/total > 25\%) } \\
\hline Yes & 8 & $1.639(1.298,2.069)$ & $<0.001$ & $<0.001,71.8 \%$ & \\
\hline No & 15 & $1.976(1.651,2.364)$ & $<0.001$ & $<0.001,75.3 \%$ & \\
\hline \multicolumn{6}{|c|}{ Tumor stage $(I I I+\mid V /$ total > 80\%) } \\
\hline Yes & 9 & $1.681(1.377,2.053)$ & $<0.001$ & $<0.001,78.0 \%$ & \\
\hline No & 8 & $1.352(1.265,1.445)$ & $<0.001$ & $0.065,47.4 \%$ & \\
\hline \multicolumn{6}{|l|}{ Detection method } \\
\hline ELISA & 7 & $2.144(1.466,3.134)$ & $<0.001$ & $<0.001,74.4 \%$ & \\
\hline latex assay & 4 & $1.513(1.266,1.810)$ & $<0.001$ & $0.026,67.6 \%$ & \\
\hline immunoturbidimetry assay & 10 & $1.363(1.264,1.470)$ & $<0.001$ & $0.065,44.1 \%$ & \\
\hline \multicolumn{6}{|l|}{ Surgery } \\
\hline Non-surgical & 8 & $2.380(1.78,3.18)$ & $<0.001$ & $0.019,58.2 \%$ & \\
\hline surgical & 4 & $1.910(1.17,3.14)$ & $<0.001$ & $0.008,74.5 \%$ & \\
\hline mixed & 5 & $1.688(1.333,2.139)$ & $<0.001$ & $0.020,65.6 \%$ & \\
\hline PFS & 7 & $1.385(1.169,1.641)$ & $<0.001$ & $0.003,69.1 \%$ & \\
\hline \multicolumn{6}{|l|}{ location } \\
\hline Asia & 6 & $1.461(1.148,1.861)$ & 0.002 & $0.007,68.5 \%$ & \\
\hline Europe & 1 & $1.340(1.167,1.539)$ & $<0.001$ & & \\
\hline \multicolumn{6}{|c|}{ Histology type (adenocarcinoma/total > 25\%) } \\
\hline Yes & 1 & $1.340(1.167,1.539)$ & $<0.001$ & & \\
\hline No & 6 & $1.461(1.148,1.861)$ & 0.002 & $0.007,68.5 \%$ & \\
\hline \multicolumn{6}{|c|}{ Tumor stage $(I I I+\mid V /$ total $>80 \%)$} \\
\hline Yes & 3 & $1.431(1.022,2.004)$ & 0.037 & $0.005,81.1 \%$ & \\
\hline No & 1 & $2.400(1.413,4.078)$ & 0.001 & & \\
\hline \multicolumn{6}{|l|}{ Detection method } \\
\hline latex assay & 1 & $1.340(1.167,1.539)$ & $<0.001$ & & \\
\hline immunoturbidimetry assay & 6 & $1.461(1.148,1.861)$ & 0.002 & $0.007,68.5 \% \%$ & \\
\hline \multicolumn{6}{|l|}{ Surgery } \\
\hline Non-surgical & 5 & $1.310(1.076,1.596)$ & 0.007 & $0.074,53.0 \%$ & \\
\hline mixed & 1 & $2.400(1.413,4.078)$ & 0.001 & & \\
\hline
\end{tabular}

patients some experts found that the levels of D-dimer can predict deep vein thrombosis (DVT) [38].These studies found that high level D-dimer may be related to the poor prognosis in tumor patients. Meanwhile, Heit found that tumor cells can promote coagulation system, increase platelet activity and damage vascular endothelial cells [39]. Another research found that activation of the blood coagulation system associated with invasive and migration biological behavior of tumors [40]. In terms of mechanism research, it may be associated with tissue factors, coagulants, colony-stimulating factors, coagulants, inflammatory cytokines, platelet activation markers were produced by tumor cells, These factors can activate the coagulation system and trigger the coagulation cascade through different signaling pathways [41-47]. However, the underlying mechanisms that why elevated D-dimer is related to a poor survival in patients with lung cancer are unknown and need further study.

With regard to clinical significance of D-dimer in lung cancer, whether anticoagulant treatment before cancerrelated treatment in lung cancer patients can change their prognosis, and whether D-dimer is associated with clinical staging and tumor tissue type are uncertain. Moreover, the value of $\mathrm{D}$-dimer as prognostic outcomes 

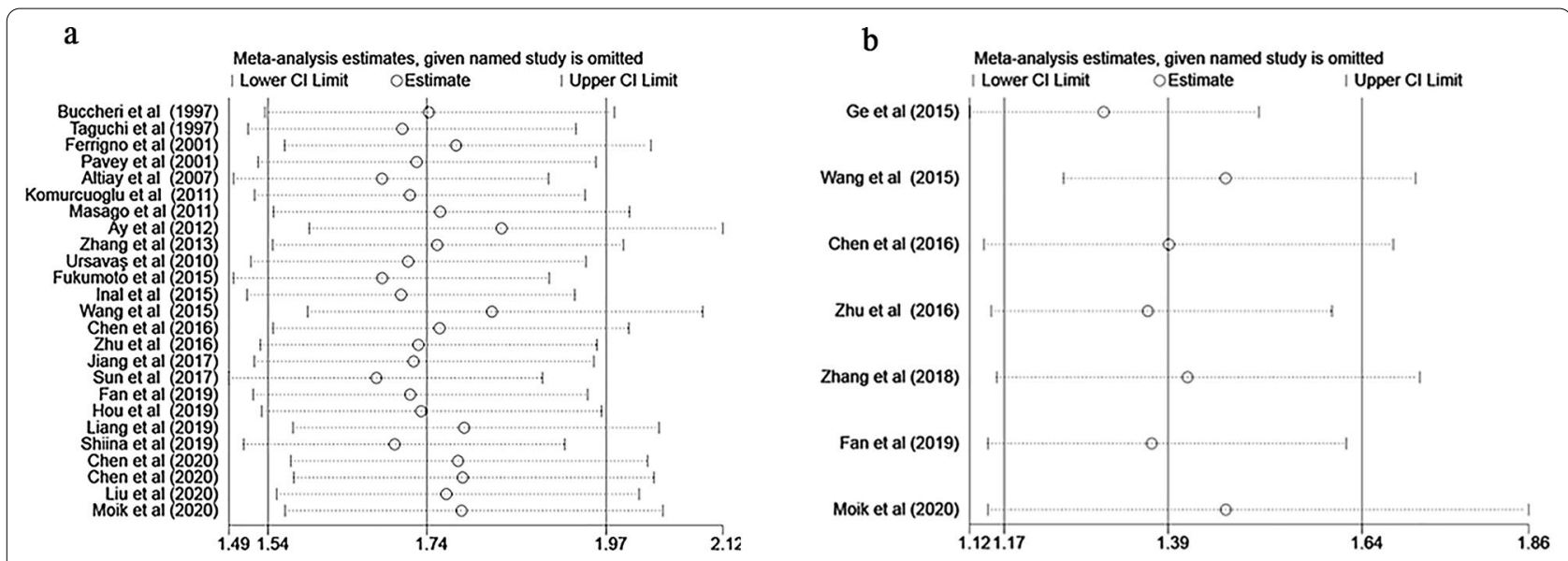

Fig. 3 The sensitivity analysis of OS (a) and PFS (b)

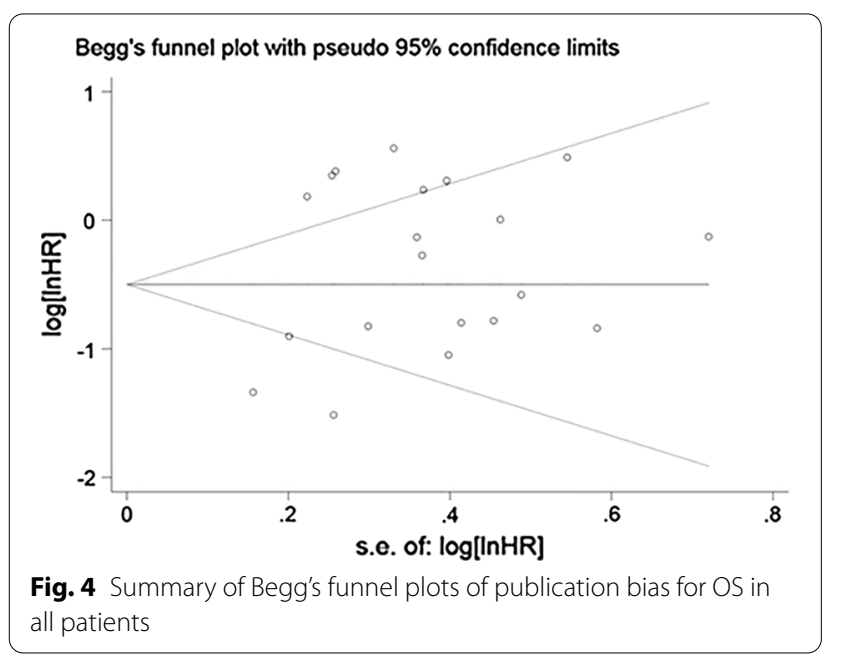

is unclear, especially in patients undergoing radiotherapy and chemotherapy. Therefore, our meta-analysis needs to approve the prognostic value of D-dimer for lung cancer.

Our meta-analysis investigated the relationship between plasma $\mathrm{D}$-dimer and prognosis in lung cancer patients. The results revealed that D-dimer as an effective coagulation factor has the potential to be a promising biomarker to predict prognosis in lung cancer patients, which could promote doctors' anticoagulant therapy selection. In our study, we estimated the significance of plasma D-dimer levels in lung cancers. Compared with these patients who have a normal plasma D-dimer, patients with a high level of plasma D-dimer have a worse overall survival prognosis ( $\mathrm{HR}=1.742$, 95\% CI:1.5421.969; $P<0.001, \mathrm{I}^{2}=72.1 \%$ ). We also found that elevated plasma D-dimer is a risk factor of PFS in lung cancers according to pooled HR (HR=1.385, 95\% CI:1.1691.641; $\left.P=0.003, \mathrm{I}^{2}=69.1 \%\right)$. Hence, we think that this meta-analysis can confirm the prognostic significance of plasma D-dimer level in lung tumors.

We concentrated on subgroup analysis of detection method revealed that studies based on the group of ELISA. We found that the HR ratio in the ELISA group was higher than that in the other two groups. In recent years, ELISA, latex method and immune turbidimetry are mainly used to detect D-dimer. However, compared with the other two methods, ELISA has higher sensitivity and specificity, so many hospitals regard ELISA as the gold standard for the detection of D-dimer. However, it takes a lot of time to use ELISA detection, so there is an urgent need for new methods to help reduce the diagnosis time. In addition, the immunoturbidimetry assay group had a lower heterogeneity $\left(\mathrm{HR}=1.363,95 \% \mathrm{CI}: 1.264,1.470, \mathrm{I}^{2}=44.1 \%\right)$, which might be the possible sources of heterogeneity.

Though fibrinolytic and coagulation activation systems have been reported to boost tumor growth through different mechanisms, including promoting angiogenesis, suppressing apoptosis of tumor cells, the mechanism association between the plasma D-dimer level and the aggressiveness of lung cancer remains unclear. Shiina et al. found that tumors in the high D-dimer group frequently invaded vessels [48]. Our subgroup analysis about tumor stage (III + IV/total $>80 \%$ ) finding high D-dimer level had a worse prognosis of OS and PFS, indicating that $\mathrm{D}$-dimer levels are associated with tumor invasion metastasis. Although high D-dimer levels might be lead to vessel injury due to tumor aggressiveness, the connection between the plasma D-dimer level and vessel invasion is unclear, and more detailed research is needed. Many studies believe that $\mathrm{HR}>2$ indicates a prognostic biomarker in practical setting [49]. When subgroup analysis of OS was performed according to different treatment methods, the OS HR of non-surgical patients was 
higher than 2. This indicates that D-dimer has a stronger predictive value in non-surgical patients, which is consistent with the following report that progression-free survival was significantly worse in patients with high D-dimer levels than normal levels at different time points before and after chemotherapy [20].

Higher heterogeneity was found in our meta-analysis for OS and PFS of the prognostic role of D-dimer $\left(P<0.001, \mathrm{I}^{2}=72.1 \%, P=0.003, \mathrm{I}^{2}=69.1 \%\right)$. To remove these biases, subgroup analyses were performed by the different nations, detecting methods, disease stage, histological type, and different treatments. When we grouped the analysis into III $+\mathrm{IV} /$ total $<80 \%$ groups, immunoturbidimetry assay group, and Europe group, the heterogeneity for OS could shrink to $47.4 \%, 44.1 \%$, and 0 . Heterogeneity in other subgroups can be reduced or eliminated accordingly.

Several limitations should be addressed in our metaanalysis. First, our meta-analysis could only include retrospective cohort studies with limited sample size and patient selection bias for analysis. As a result, the statistical power of our meta-analysis could be decreased. Second, the main outcomes could not be fully obtained from all these original studies, and PFS cannot be tested for publication bias due to insufficient data. Moreover, D-dimer levels were measured only once which may result in errors. In conclusion, more prospective research with larger sample size is executed to confirm the further relationship between preoperative D-dimer molecular markers and prognostic characteristics.

\section{Conclusions}

We conducted this meta-analysis revealed that the high plasma D-dimer level leads to lower survival than in the low D-dimer level, which might provide an important clue for high plasma D-dimer level as an independent factor of poor prognosis in patients with lung cancer. In the future, more prospective cohort studies are needed to study and verify.

\section{Abbreviations}

VTE: Venous thromboembolism; SCLC: Small cell lung cancer; TNM: Tumor node metastasis; DFS: Disease-free survival; OS: Overall survival; RFS: Relapsefree survival; HR: Hazard ratio; Cl: Confidence interval; ELISA: Enzyme-linked immunosorbent assay.

\section{Supplementary Information}

The online version contains supplementary material available at https://doi. org/10.1186/s13019-021-01618-4.

Additional file 1 Estimated HR summary for a OS in patients with the detection method of immunoturbidimetry,b OS in patients with the detection method of latex assay, c OS with the detection method of ELISA
Additional file 2 Estimated HR summary for a PFS in patients with the detection method of immunoturbidimetry,b PFS in patients with the detection method of latex assay

Additional file 3 Estimated HR summary for a OS in patients with the Histological type of $>25 \%$ adenocarcinoma,b OS in patients with the Histological type of $<25 \%$ adenocarcinoma

Additional file 4 Estimated HR summary for a PFS in patients with the Histological type of $>25 \%$ adenocarcinoma,b PFS in patients with the Histological type of $<25 \%$ adenocarcinoma

Additional file $\mathbf{5}$ Estimated HR summary for a OS in patients with the Disease stage of stage III-IV/total $>80 \%, \mathrm{~b}$ OS in patients with the Disease stage of stage III-IV/total $<80 \%$ Estimated HR summary for a OS in patients with the Disease stage of stage III-IV/total $>80 \%, \mathrm{~b}$ OS in patients with the Disease stage of stage III-IV/total $<80 \%$

Additional file 6 Estimated HR summary for a PFS in patients with the Disease stage of stage III-IV/total $>80 \%, \mathrm{~b}$ PFS in patients with the Disease stage of stage III-IV/total $<80 \%$

Additional file 7 Estimated HR summary for a OS in patients in Asia countries,b OS in patients in Europe countries,c OS in patients in Oceania countries

Additional file 8 Estimated HR summary for a PFS in patients in Asia countries,b PFS in patients in Europe countries

Additional file 9 Estimated HR summary for a OS in patients with non-surgical,b OS in patients with surgical,c OS in patients with mixed treatments

Additional file 10 Estimated HR summary for a PFS in patients with nonsurgical,b PFS in patients with mixed treatments

\section{Acknowledgements}

Not applicable.

\section{Authors' contributions}

MM put forward the idea, RC designed the research; All authors contributed to literature searching, data extraction and analysis; MM wrote the manuscript: $L Y$ revised the manuscript; $M M$ and $R C$ contributed equally to this manuscript. The authors approved the final version of the manuscript.

\section{Funding}

This research is supported by the following funds: National Natural Science Foundation of China (No. 81860325) and High-level health technical personnel of Yunnan Provincial Health Commission (No. L-2017006).

\section{Availability of data and materials}

The datasets generated and analyzed during the current study are available from the corresponding author on reasonable request.

\section{Declarations}

\section{Ethics approval and consent to participate}

All analyses were based on previous published studies, thus no ethical approval and patient consent are required.

Consent for publication

Not applicable.

\section{Competing interests}

All authors declare no conflict of interest with respect to research, authorship and publication of the article.

\section{Author details}

${ }^{1}$ Department of Thoracic Surgery, The Third Affiliated Hospital of Kunming Medical University, No. 519 Kunzhou Road, Xishan District, Kunming City, Yunnan Province, China. ${ }^{2}$ Department of Cardiothoracic Surgery, The Sixth Affiliated Hospital of Kunming Medical University, Yuxi, China. 
Received: 16 June 2021 Accepted: 11 August 2021

Published online: 28 August 2021

\section{References}

1. Ay C, Dunkler D, Pirker R, Thaler J, Quehenberger P, Wagner O, et al. High $\mathrm{D}$-dimer levels are associated with poor prognosis in cancer patients. Haematologica. 2012;97(8):1158.

2. Tas F, Kilic L, Serilmez M, Keskin S, Sen F, Duranyildiz DJRM. Clinical and prognostic significance of coagulation assays in lung cancer. Respirat Med. 2013;107(3):451-7.

3. Hisada Y, Mackman NJB. Cancer-associated pathways and biomarkers of venous thrombosis. J Am Soc Hematol. 2017:130(13):1499-506.

4. Louneva N, Maity A. Plasma D-dimer levels are elevated in radiation oncology patients. Kennedy ARJRr. 2020;193(1):46-53.

5. Schorling RM, Pfrepper C, Golombek T, Cella CA, Muñoz-Unceta N, Siegemund $\mathrm{R}$, et al. Evaluation of biomarkers for the prediction of venous thromboembolism in ambulatory cancer patients. Oncol Res Treatm. 2020:43(9):412-21.

6. Kilıc M, Yoldas O, Keskek M, Ertan T, Tez M, Gocmen E, et al. Prognostic value of plasma D-dimer levels in patients with colorectal cancer. Colorectal Dis. 2008:10(3):238-41.

7. Liu L, Zhang X, Yan B, Gu Q, Zhang X, Jiao J, Yue X. Elevated plasma $D$-dimer levels correlate with long term survival of gastric cancer patients. PloS one. 2014;9(3):e9054.

8. Lin Y, Liu Z, Qiu Y, Zhang J, Wu H, Liang R, Zou D. Clinical significance of plasma D-dimer and fibrinogen in digestive cancer: a systematic review and meta-analysis. Eur J Surg Oncol. 2018;44(10):1494-503.

9. Inal T, Anar C, Polat G, Unnsal I, Halilçolar H. The prognostic value of D-dimer in lung cancer. Clin Respirat J. 2015:9(3):305-13.

10. Altiay G, Ciftci A, Demir M, Kocak Z, Sut N, Tabakoglu E, Caglar T. High plasma D-dimer level is associated with decreased survival in patients with lung cancer. Clin Oncol. 2007;19(7):494-8.

11. Falanga A, Marchetti M, Vignoli A. Coagulation and cancer: biological and clinical aspects. J Thrombosis Haemostasis. 2013;11(2):223-33.

12. Tierney JF, Stewart LA, Ghersi D, Burdett S, Sydes MR. Practical methods for incorporating summary time-to-event data into meta-analysis. Trials. 2007;8(1):1-16.

13. Guyot P, Ades AE, Ouwens MJ, Welton NJ. Enhanced secondary analysis of survival data: reconstructing the data from published Kaplan-Meier survival curves. BMC Med Res Methodol. 2012:12(1):1-13.

14. Higgins JP, Thompson SG. Quantifying heterogeneity in a meta-analysis. Stat Med. 2002;21(11):1539.

15. Liu Q, He J, Ning R, Tan L, Zeng A, Zhou S. Pretreatment plasma d-dimer levels as an independent prognostic factor for overall survival among patients with advanced non-small-cell lung cancer. J Int Med Res. 2020;48(10):0300060520962661

16. Ferrigno D, Buccheri G, Ricca I. Prognostic significance of blood coagulation tests in lung cancer. Eur Respirat J. 2001;17(4):667-73.

17. Buccheri $G$, Torchio $P$, Ferrigno D. Plasma levels of $D$-dimer in lung carcinoma: clinical and prognostic significance. Cancer. 2003;97(12):3044-52.

18. Chen Y, Yu H, Wu C, Li J, Jiao S, Hu Y, Li A. Prognostic value of plasma D-dimer levels in patients with small-cell lung cancer. Biomed Pharmacotherap. 2016;81:210-7.

19. Zhang PP, Sun JW, Wang XY, Liu XM, Li K. Preoperative plasma D-dimer levels predict survival in patients with operable non-small cell lung cancer independently of venous thromboembolism. Eur J Surg Oncol (EJSO). 2013;39(9):951-6

20. Ge LP, Li J, Bao QL, Chen P, Jiang Q, Zhu LR. Prognostic and predictive value of plasma D-dimer in advanced non-small cell lung cancer patients undergoing first-line chemotherapy. Clin Transl Oncol. 2015;17(1):57-64.

21. Wang $Y$, Wang Z. Predictive value of plasma D-dimer levels in patients with advanced non-small-cell lung cancer. OncoTargets therapy. 2015;8:805

22. Zhu $L R$, Li J, Chen $P$, Jiang $Q$, Tang XP. Clinical significance of plasma fibrinogen and D-dimer in predicting the chemotherapy efficacy and prognosis for small cell lung cancer patients. Clin Transl Oncol. 2016;18(2):178-88.
23. Jiang $X$, Mei $X, W u H$, Chen X. D-dimer level is related to the prognosis of patients with small cell lung cancer. Ann Transl Med 2017;5(20): 394

24. Zhang C, Jia Y, Jia Y, Zhang X, Li K. Prognostic and predictive value of plasma D-dimer levels in patients with small-cell lung cancer. Int J Clin Oncol. 2018;23(6):1070-5.

25. Fan S, Zhao G, An G. High pretreatment plasma D-dimer levels are associated with shorter overall survival in patients with small cell lung cancer. J Int Med Res. 2019;47(1):215-24.

26. Hou C, Jiang F, Ma H, Zhu Q, Wang Z, Zhao B, Li J. Prognostic role of preoperative platelet, fibrinogen, and D-dimer levels in patients with non-small cell lung cancer: A multicenter prospective study. Thoracic Cancer, 2019;10(2), 304-311.

27. Liang HG, Gao K, Jia R, Li J, Wang C. Prognostic significance of the combination of preoperative fibrinogen and the neutrophillymphocyte ratio in patients with nonsmall cell lung cancer following surgical resection. Oncol Lett. 2019;17(2):1435-44.

28. Buccheri G, Ferrigno D, Ginardi C, Zuliani C. Haemostatic abnormalities in lung cancer: prognostic implications. Eur J Cancer. 1997;33(1):50-5.

29. Ursavaş A, Karadağ M, Uzaslan E, Yesilkaya S, Coşkun F, Eğe E. Prognostic significance of plasma D-dimer levels in patients with lung cancer. Eur J Gen Med. 2010;7(2):155-60

30. Moik F, Zöchbauer-Müller S, Posch F, Pabinger I, Ay C. Systemic Inflammation and Activation of Haemostasis Predict Poor Prognosis and Response to Chemotherapy in Patients with Advanced Lung Cancer. Cancers. 2020;12(6):1619.

31. Chen C, Li J, Li J, Wang X, Wang X, Du N, et al. Application of an elevated plasma D-dimer cut-off value improves prognosis prediction of advanced non-small cell lung cancer. 2020;8(18).

32. Taguchi O, Gabazza EC, Yasui H, Kobayashi T, Yoshida M, Kobayashi $\mathrm{H}$. Prognostic significance of plasma D-dimer levels in patients with lung cancer. Thorax. 1997:52(6):563-5.

33. Pavey SJ, Hawson GAT, Marsh NA. Impact of the fibrinolytic enzyme system on prognosis and survival associated with non-small cell lung carcinoma. Blood Coagul Fibrinol. 2001;12(1):51-8.

34. Altiay G, Ciftci A, Demir M, Kocak Z, Sut N, Tabakoglu E, Caglar T. High plasma D-dimer level is associated with decreased survival in patients with lung cancer. Clin Oncol. 2007;19(7):494-8.

35. Komurcuoglu B, Ulusoy S, Gayaf M, Guler A, Ozden E. Prognostic value of plasma D-dimer levels in lung carcinoma. Tumori J. 2011;97(6):743-8.

36. Masago K, Fujita S, Mio T, Togashi Y, Kim YH, Hatachi Y, Mishima M. Clinical significance of the ratio between the alpha 2 plasmin inhibitor-plasmin complex and the thrombin-antithrombin complex in advanced nonsmall cell lung cancer. Med Oncol, 2011;28(1), 351-356

37. Han D, Hartaigh B, Lee JH, Cho IJ, Shim CY, Chang HJ, Chung N. Impact of D-dimer for prediction of incident occult cancer in patients with unprovoked venous thromboembolism. PLoS One, 2016;11(4), e0153514

38. Fei X, Wang H, Yuan W, Wo M, Jiang L. Tissue Factor Pathway Inhibitor-1 Is a Valuable Marker for the Prediction of Deep Venous Thrombosis and Tumor Metastasis in Patients with Lung Cancer. BioMed research international. 2017;2017:8983763. PubMed PMID: 28246607. Pubmed Central PMCID: PMC5299162. Epub 2017/03/02. eng.

39. Heit JA. Cancer and venous thromboembolism: scope of the problem. Cancer control : journal of the Moffitt Cancer Center. 2005 Sep;12 Suppl 1:5-10. PubMed PMID: 16179898. Epub 2005/09/24. eng.

40. Beer JH, Haeberli A, Vogt A, Woodtli K, Henkel E, Furrer T, et al. Coagulation markers predict survival in cancer patients. Thrombosis and haemostasis. 2002 Nov;88(5):745-9. PubMed PMID: 12428088. Epub 2002/11/13. eng.

41. Fernandes CJ, Morinaga LTK, Alves JLJ, Castro MA, Calderaro D, Jardim CVP, et al. Cancer-associated thrombosis: the when, how and why. European respiratory review : an official journal of the European Respiratory Society. 2019 Mar 31;28(151). PubMed PMID: 30918022. Epub 2019/03/29. eng.

42. Falanga A, Marchetti M. Venous thromboembolism in the hematologic malignancies. Journal of clinical oncology : official journal of the American Society of Clinical Oncology. 2009 Oct 10;27(29):4848-57. PubMed PMID: 19752334. Epub 2009/09/16. eng.

43. Piccioli A, Falanga A, Baccaglini U, Marchetti M, Prandoni P. Cancer and venous thromboembolism. Seminars in thrombosis and hemostasis. 2006 Oct;32(7):694-9. PubMed PMID: 17024596. Epub 2006/10/07. eng. 
44. Hisada Y, Mackman N. Mouse models of cancer-associated thrombosis. Thrombosis research. 2018 Apr;164 Suppl 1(Suppl 1):S48-s53. PubMed PMID: 29306575. Pubmed Central PMCID: PMC5942599. Epub 2018/01/08. eng.

45. Riedl J, Preusser M, Nazari PM, Posch F, Panzer S, Marosi C, et al. Podoplanin expression in primary brain tumors induces platelet aggregation and increases risk of venous thromboembolism. Blood. 2017 Mar 30;129(13):1831-9. PubMed PMID: 28073783. Pubmed Central PMCID: PMC5823234. Epub 2017/01/12. eng.

46. Sharma BK, Flick MJ, Palumbo JS. Cancer-Associated Thrombosis: A TwoWay Street. Seminars in thrombosis and hemostasis. 2019 Sep;45(6):55968. PubMed PMID: 31382306. Epub 2019/08/06. eng.

47. Gil-Bernabé AM, Ferjancic S, Tlalka M, Zhao L, Allen PD, Im JH, et al. Recruitment of monocytes/macrophages by tissue factor-mediated coagulation is essential for metastatic cell survival and premetastatic niche establishment in mice. Blood. 2012 Mar 29;119(13):3164-75. PubMed PMID: 22327225. Epub 2012/02/14. eng.

48. Shiina Y, Nakajima T, Yamamoto T, Tanaka K, Sakairi Y, Wada H, et al. The D-dimer level predicts the postoperative prognosis in patients with nonsmall cell lung cancer. 2019;14(12):e0222050.

49. Hayes DC, Secrist H, Bangur CS, Wang T, Zhang X, Harlan D, Zehentner BK. Multigene real-time PCR detection of circulating tumor cells in peripheral blood of lung cancer patients. Anticancer Res. 2006;26(2B):1567-75.

\section{Publisher's Note}

Springer Nature remains neutral with regard to jurisdictional claims in published maps and institutional affiliations.
Ready to submit your research? Choose BMC and benefit from:

- fast, convenient online submission

- thorough peer review by experienced researchers in your field

- rapid publication on acceptance

- support for research data, including large and complex data types

- gold Open Access which fosters wider collaboration and increased citations

- maximum visibility for your research: over 100M website views per year

At BMC, research is always in progress.

Learn more biomedcentral.com/submissions 